\title{
Stretchable Conductive Composites Based on Metal Wools for Use as Electrical Vias in Soft Devices
}

\section{Citation}

Lessing, Joshua, Stephen A. Morin, Christoph Keplinger, Alok S. Tayi, and George M. Whitesides. 2015. "Stretchable Conductive Composites Based on Metal Wools for Use as Electrical Vias in Soft Devices." Advanced Functional Materials 25 (9) (January 21): 1418-1425. doi:10.1002/ adfm. 201403396.

\section{Published Version}

doi:10.1002/adfm.201403396

\section{Permanent link}

http://nrs.harvard.edu/urn-3:HUL.InstRepos:24981606

\section{Terms of Use}

This article was downloaded from Harvard University's DASH repository, and is made available under the terms and conditions applicable to Open Access Policy Articles, as set forth at http:// nrs.harvard.edu/urn-3:HUL.InstRepos:dash.current.terms-of-use\#OAP

\section{Share Your Story}

The Harvard community has made this article openly available.

Please share how this access benefits you. Submit a story.

\section{Accessibility}


DOI: 10.1002/XXXXX

Article type: Full Paper

Stretchable Conductive Composites Based on Metal Wools for Use as Electrical Vias in Soft Devices

Joshua Lessing ${ }^{l}$, Stephen A. Morin ${ }^{l}$, Christoph Keplinger ${ }^{l}$, Alok S. Tayi ${ }^{l}$, and George M.

Whitesides $^{1,2, *}$

[*] Prof. G. M. Whitesides

${ }^{1}$ Department of Chemistry and Chemical Biology, Harvard University, 12 Oxford Street, Cambridge, MA 02138, (USA).

${ }^{2}$ Wyss Institute for Biologically Inspired Engineering, Harvard University, 3 Blackfan Circle, Boston, MA 02115, (USA).

E-mail: gwhitesides@gmwgroup.harvard.edu

Dr. J. Lessing, Dr. S.A. Morin, Dr. C. Keplinger, Dr. A.S. Tayi

${ }^{1}$ Department of Chemistry and Chemical Biology, Harvard University, 12 Oxford Street, Cambridge, MA 02138, (USA).

Keywords: conductive elastomers, stretchable composites, soft robotics, soft devices, metal wools 


\title{
WILEY-VCH
}

\begin{abstract}
Soft devices can be bent, stretched and compressed reversibly, but conventional wires are rigid. This work describes stretchable composites that are easily fabricated with simple tools and commodity materials, and that can provide a strategy for electrical wiring that meets certain needs of soft devices. These composites are made by combining metal wool and elastomeric polymers. Embedding fine (average fiber width $\sim 25 \mu \mathrm{m}$ ) steel wool (or other metal wools) in a silicone polymer creates an electrically conductive path through the nonconductive elastomer. This composite is flexible, stretchable, compressible, inexpensive, and simple to incorporate into the bodies of soft devices. It is also electrically anisotropic, and shows maximum conductivity along the majority axis of the fibers, but maximum extension perpendicular to this axis. The utility of this composite for creating an electrically conductive path through an elastomer was demonstrated in several devices, including: a soft, solderless breadboard, a soft touch sensor, and a soft strain gauge.
\end{abstract}

\section{Introduction}

Soft devices are machines built from soft materials (e.g. elastomers, gels, liquids). ${ }^{[1,2]}$ These soft devices are useful for their ability to change their size and shape readily upon electrical,${ }^{[3-5]}$ chemical,${ }^{[6]}$ pneumatic,${ }^{[2,7,8,9]}$ ferrofluidic,${ }^{[10]}$ or hydraulic ${ }^{[9]}$ actuation. In addition, the low stiffness of the materials used to construct these devices (Young's modulus $<$ $10 \mathrm{MPa}$ ) enables them to deform readily in response to external forces. These attributes allow soft devices to perform functions that are challenging for hard machines; examples include interacting with delicate, soft materials (e.g. biological tissues ${ }^{[11]}$ ), and performing unstructured tasks (e.g. gripping objects of undefined shape ${ }^{[2]}$ ). Integrating electronics for control systems 


\section{WILEY-VCH}

and sensors, or other electrical functions into soft devices will be an important step in their evolution.

Metal wires, used in conventional electronics, when embedded in a soft device, often delaminate from the surrounding soft materials, or break, when the device bends and stretches, as it would during operation. We wished to have a stretchable, highly conductive material that would be inexpensive and simple to fabricate and use, and that could be formed into conductive paths to carry and distribute electrical potential and current in soft machines.

We accomplished this goal — albeit using a solution with advantages and disadvantages by embedding a highly extensible network of metal fiber — a metal "wool" — in a highly extensible elastomer to create conductive elastomeric composites (CEC), and used these composites to make simple devices that took advantage of their properties. These properties are particularly useful: i) flexible conductivity. CECs allow for conductive contact to be made and maintained between the CEC and other materials (e.g., metal surfaces, wires, liquids, electrical components etc.), even if the device in which the CEC is embedded is moving and changing shape. ii) conformability. CECs can conform to the shape of a range of surfaces, and do not require standardized plugs to enable the formation of electrical connections. iii) low cost. CECs can be made from commodity materials to minimize the cost of prototyping and manufacturing.

Elastomeric materials present new opportunities for building soft devices, but also present technical challenges. A CEC embedded in a soft device can maintain the flow of electrical current while the soft device simultaneously and reversibly changes shape, surface area, and thickness. They are not conventional wires - structures appropriate for small devices - nor are they flexible conductive polymers. They are probably closer in spirit to a braided metal 


\section{WILEY-VCH}

cable, but are more elastomeric. Most importantly, they can be fashioned easily and inexpensively into stretchable conductive macroscopic slabs that can be readily incorporated into soft devices, using commodity materials and simple tools. CECs thus surmount some of the technical challenges of stretchable electrical devices, and can therefore satisfy the need for certain types of wires in soft devices, and do so in ways that have no exact counterpart with conventional wiring.

\section{Background}

Stretchable electronics. A wide variety of soft embodiments of common electronic devices (e.g. transistors, ${ }^{[12,13]}$ displays, ${ }^{[14,15]}$ batteries, ${ }^{[16,17]}$ electromechanical transducers, ${ }^{[3]}$ speakers, ${ }^{[4]}$ thermal sensors, ${ }^{[18]}$ strain sensors, ${ }^{[19]}$ pressure sensors, ${ }^{[18,19]}$ and photo detectors ${ }^{[20]}$ ) have been successfully fabricated. To meet the electrical needs of these devices, an array of stretchable electrical conduits for supplying electrical potential and current have been created. For example, liquid metals have been used to create stretchable wires by embedding channels filled with EGaIn into elastomers to achieve highly conductive structures. ${ }^{[21]}$ Metal ion implantation can also be used to create stretchable conductive structures requiring either the use of a plasma chamber ${ }^{[22]}$ or multiple, wet-chemistry, processing steps. ${ }^{[23]}$ One mechanical approach is to create conductive, net-shaped, structures out of relatively inelastic materials using mechanical processing with cutting tools to convert ridged substrates for electrical components (e.g. polyimide sheets) into stretchable nets, ${ }^{[15,18]}$ although they have also been fabricated via molding of polydimethylsiloxane (PDMS). ${ }^{[2]}$ Recently, hydrogels infused with ionic conductors have been used to create transparent, biocompatible, stretchable devices, but these materials cannot be used to supply continuous DC currents, or high AC currents. ${ }^{[4]}$ One of the 


\section{WILEY-VCH}

oldest and most thoroughly investigated approaches to making stretchable conductors is to create composites, incorporating conductive particles and elastomers, which rely on a percolation network to conduct electricity. These materials are also not appropriate replacements for conventional wires since their resistivity is typically large, and can change by several orders of magnitude during reorganization of the percolation network induced by mechanical strain. ${ }^{[14,20 \text {, }}$ 25] To date, the most successful approaches to creating small, stretchable wires — utilized in the work of Wagner, Rogers, and others — have used lithographic techniques to create serpentine patterns of metal films on unstrained elastomers ${ }^{[17,26]}$ or on pre-strained elastomers to make buckled metal films. ${ }^{[13,27]}$ These approaches have enabled the creation of wires capable of sustaining high strains (typically $>30 \%$ with strains $>300 \%$ having been reported for serpentine patterns ${ }^{[17]}$ ). Our composites are conceptuality similar to conductive nets, serpentine interconnects, and buckled films because they rely on the bending of thin filaments of rigid materials to create an effective elasticity. In contrast to these structures, our composites are macroscopic, and designed with a focus on creating an inexpensive and easily fabricated conductive path through an elastomeric material that will satisfy some of the needs in the field of soft machines.

Metal wools are a mat of metal filaments machined from a metal rod. They are inexpensive and commercially available in a variety of metals (e.g. steel, copper, aluminum, brass), and are usually sold in bulk as rolls or pads. The most common metal wool is steel wool. This material is commonly used for scouring, polishing, filtration, and acoustic dampening. Steel fibers are used in composites for structural reinforcement (e.g. concrete, cement, and rubber tires ${ }^{[28]}$ ), and to improve the thermal conductivity and wear resistance of an elastomeric friction material (e.g. brake pads ${ }^{[29]}$ ). To our knowledge, composites consisting of 


\section{WILEY-VCH}

steel wools embedded in elastomers have not previously been used to create stretchable, electrical connections.

\section{Experimental Design}

Steel is a poor conductor relative to other metals (e.g. silver, copper, gold, aluminum, etc.), making steel an unlikely material for fabricating wires. Despite steel's poor conductivity, a steel wool was used for constructing CECs because the combination of steel wool's electrical and mechanical properties is functionally useful for a stretchable conductive material: (i) as a metal, it can provide an electrically conductive path across the fiber network, (ii) as a bundled material, steel wool produces an entangled mat of spring-like fibers that bend when strained rather than fracturing, (iii) as a wool made from steel, it maximizes the reversibility of the composite upon elongation due to its higher yield strength relative to other materials ( e.g. pure copper, bronze, brass, pure aluminum, and many aluminum alloys, etc.).

As in conductive nets, serpentine interconnects, and buckled films, the stretchability of the metal wool is achieved through bending of individual thin filaments. When a bundle of metal wool fibers is stretched in a direction perpendicular to the majority fiber axis, the fibers bend away from each other. The bending stiffness of a metal wool fiber, assuming it is in the shape of a rod, is given by (Equation 1):

$$
E I=\frac{E \pi d^{4}}{64}
$$

where $E$ is the elastic modulus, $I$ is the area moment of inertia, $E I$ is the bending stiffness, and the diameter of the fiber is $d .^{[30]}$ A fine metal wool was chosen because bending stiffness scales 


\section{WILEY-VCH}

nonlinearly with the diameter of the metal fiber; fibers in a fine metal wool are dramatically more bendable than those in a coarse metal wool. We used a metal wool with an average fiber width of $\sim 25 \mu \mathrm{m}$ as our conductor to construct the CECs shown in this paper; this width allowed us to create a conductive composite capable of reversible deformation.

Our composite combines the elastic range of elastomeric polymers with the electrical conductivity of steel wool to realize a stretchable, conductive material that can be embedded in the body of a soft device. We used a silicone elastomer because of convenience (e.g. many inexpensive formulations are available with a range of mechanical and optical properties, etc.) but other elastomers (e.g., polyurethanes and natural rubbers) would also be suitable. Ecoflex 00-30 (Smooth-On, Inc.) silicone elastomer was specifically chosen for the fabrication of all CECs because it has been previously used to construct soft actuators, ${ }^{[2,7]}$ ensuring the compatibility of our CEC's with many soft robots.

\section{Results and Discussion}

\subsection{CEC Characterization}

We characterized the anisotropy in fiber orientation of the CEC polymer composite by collecting micro X-ray computed tomographic data on a CEC sheet (Figure S1A and Video S1). The Supporting Information describes the method used for fabricating this sample and all other samples presented in this paper. VGStudio software was used to measure the steel wool fiber orientation distribution in the sheet. The steel wool in the CEC had a distribution of fiber orientations centered on the long axis of the unfurled roll (which we refer to as the majority fiber axis) with an angle deviation of HWHM $=42^{\circ}$. Mechanical testing of CEC composite sheets 


\section{WILEY-VCH}

indicated they were relatively rigid along the majority fiber axis, but extensible along the axis perpendicular to the majority fiber axis (Figure S1B).

The anisotropy in the orientation of the steel fibers results in an anisotropy in the conductivity of the CEC composite (Figure 1 and Video S2). The flow of electrical current through a CEC sheet when current is transmitted parallel and perpendicular to the majority fiber axis was visualized by collecting their thermal profiles - the pattern of resistive heating that occurs when a 5.0 A current is transmitted through the CEC for 10 seconds. Figure 1A indicates that when the electrical leads are placed in line with the majority fiber axis, electrical current flows in a narrow path between the leads. Figure 1B suggests that when the electrical leads are connected perpendicularly to the axis of the majority of the fibers, the current takes a tortuous route through the $\mathrm{CEC}$ sheet. This difference in the flow of electrical current helps to explain the difference in resistance observed at the end of each measurement when transmitting current parallel, $0.6 \Omega$, and perpendicular, $1.5 \Omega$, to the majority fiber axis.

The electrical and mechanical anisotropy of the CEC material can be diminished by fabricating a layered composite with the majority fiber axis of each metal wool sheet oriented in a different direction. However, placing the metal wool sheets in multiple directions generates a rigid composite. This type of structure would be akin to the multi-layered construction used in structural engineering, where triaxial and quadraxial fibrous sheets are commonly used to create nearly isotropic rigid composites.

We measured the resistivity of a CEC sheet as it was strained (Figure 2). These measurements were obtained using a four point probe to compensate for the effect of contact resistance and the resistivity values were obtained by accounting for geometrical changes to the sample during elongation. Figure 2A illustrates how the stretchability of a CEC sheet is 
A
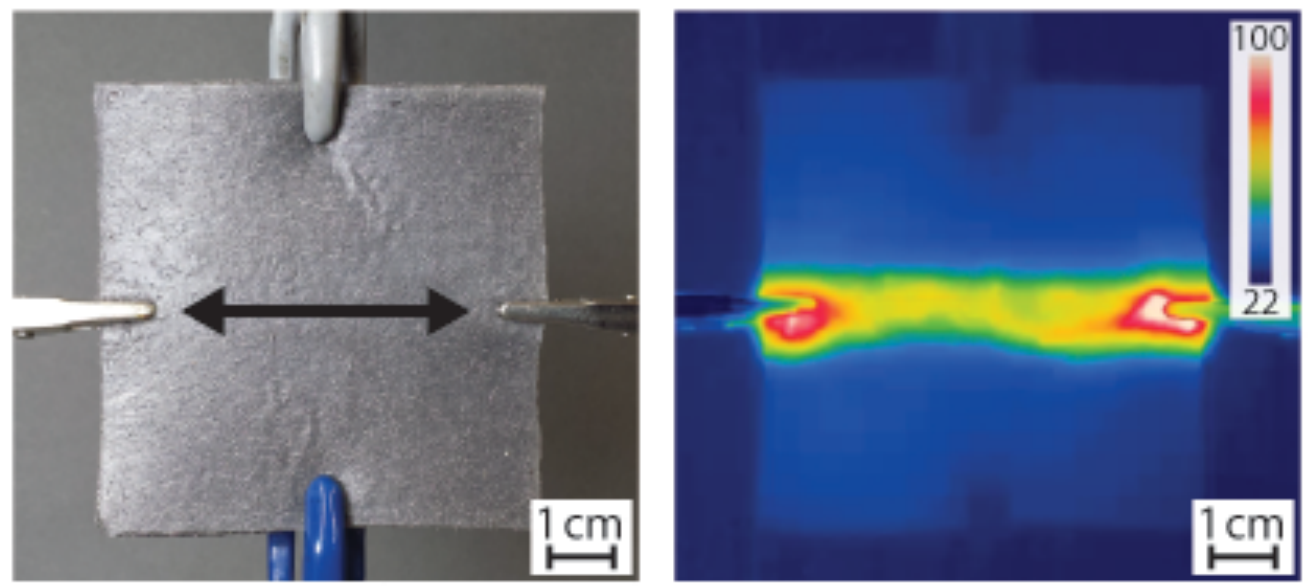

B
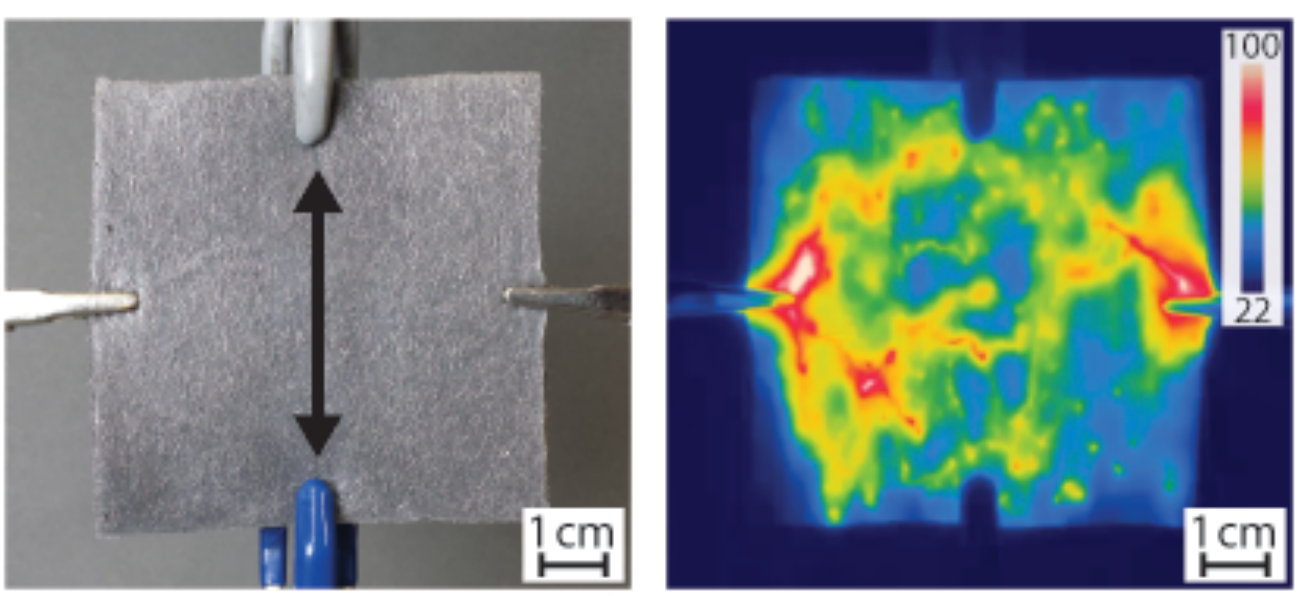

Figure 1. Characterization of Anisotropic Conductivity. The thermal signature generated by resistive heating when a current of 5.0 A passes through a CEC sheet for 10 seconds was used to illustrate the path taken by the electrical current while traversing the sheet. (A) Optical image (Left) and thermal image (Right) of a CEC sheet with electrical leads attached parallel to the majority fiber axis. (B) Optical image (Left) and thermal image (Right) of a CEC sheet with electrical leads attached perpendicular to the majority fiber axis. In the optical photographs an arrow indicates the direction of the majority fiber axis. The temperature scales shown in the thermal images are provided in degrees Celsius. 


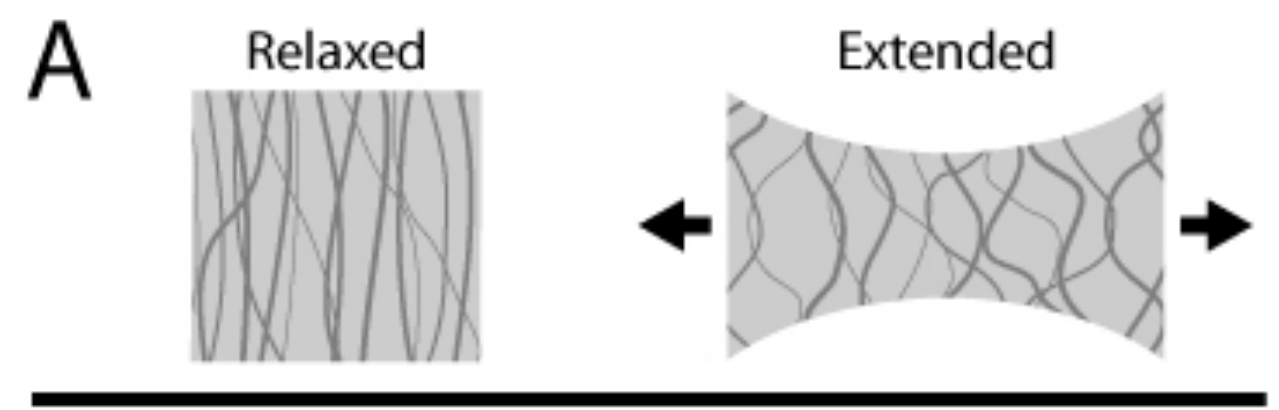

B

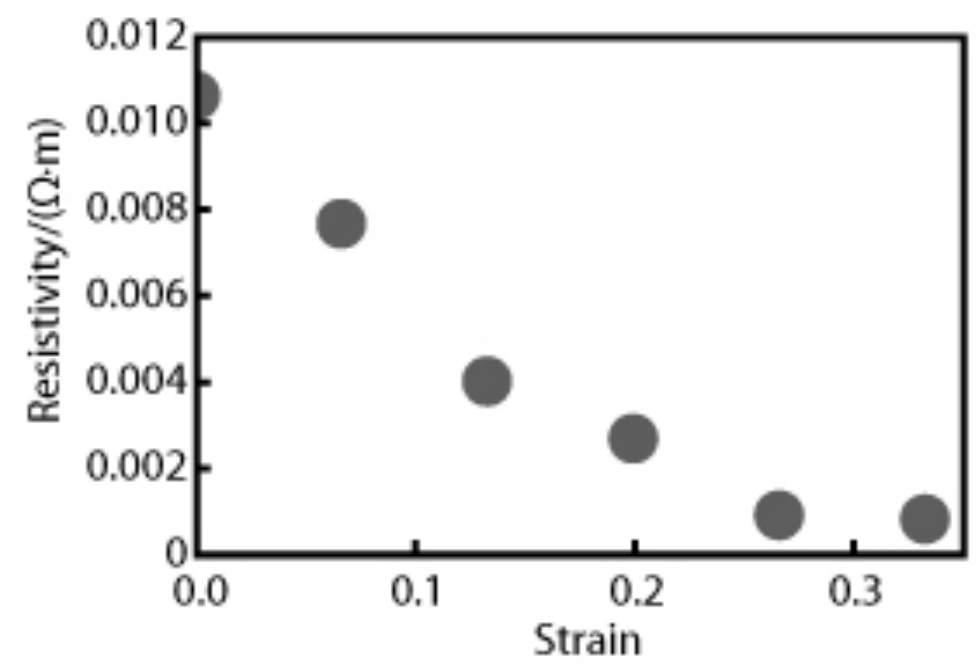

Figure 2. Materials Characterization. (A) Illustration of a CEC sheet in a relaxed and extended state. Metal wool fibers are shown in dark gray and elastomer in light gray. (B) Plot of resistivity vs. strain for a representative CEC sheet, made from steel wool and a silicone elastomer, that was elongated to a strain of 0.33 in the direction perpendicular to the majority fiber axis as current was passed in the same direction as the elongation. Strain data is provided based on measurements of engineering strain, $e=\Delta L / L$, where $e$ is the engineering strain, $L$ is the original length of the sample and $\Delta L$ is the change in the length of the sample. 


\section{WILEY-VCH}

achieved through a combination of elastically deforming the silicone polymer and bending of the thin rigid filaments of the metal wool. Figure 2B shows a plot of resistivity vs. strain for a CEC sheet, made from steel wool, which was elongated in the direction perpendicular to the majority fiber axis, as current flowed in the same direction as the elongation. The plot shows a reduction in resistivity from $1.06 \times 10^{-2}$ in its relaxed state to $0.08 \times 10^{-2} \Omega \cdot \mathrm{m}$ at a strain of 0.33 . A similar trend is also observed for CEC sheets made from aluminum and bronze wool (Figure S3). Video $\mathbf{S 3}$ demonstrates the reduction in resistance of a CEC sheet as a function of elongation; this decrease (we hypothesize) is the result of an increase in the number of points of intersection between neighboring conductive fibers as the entangled mat of fibers in the CEC is elongated. We believe that this increase in the number of electrical connections shortens the average path taken by electrons when traversing the sheet thereby reducing the resistance of the composite. The data collected for CEC sheets made from steel, aluminum, and bronze wools all show that the resistivity of a CEC sheet, in its relaxed or extended state, is higher than the resistivity of a metallic sheet of similar dimensions, but is sufficiently low that a CEC can act as an electrical connection in many circuits. Since these CEC sheets are $<1 \%$ by volume metal wool $(0.4 \pm 0.2 \%$ metal wool by volume for all steel wool sheets based on $\mathrm{n}=6$ measurements $)$ and metal wools have an inhomogeneous fiber density, it was technically challenging to fabricate samples with identical resistivity and stiffness using our current method. It was for this reason that the data presented in Figure 2, S1, and S3 and Video S3 were collected for representative samples. In the sections to follow, all CEC vias were fabricated with $2.0 \pm 0.7 \%$ steel wool by volume ( $\mathrm{n}=6$ measurements of metal wool volume). This change in composition generated CECs with consistent electrical properties at the expense of the composites extensibility. As we 


\section{WILEY-VCH}

will demonstrate, these higher metal composition composites proved useful for making electrical vias in highly extensible soft devices.

\subsection{Conducting electricity through elastomeric sheets}

CECs can be used to create an electrical path perpendicular to an elastomeric sheet.

Figure S4 shows a sheet made from a silicone elastomer with a CEC disk at its center; this disk creates an electrical pathway across the sheet with a resistance of $1.0 \Omega$. Here, the majority fiber axis of the CEC disk is oriented perpendicularly to the plane of the sheet; this orientation was chosen to minimize the resistance across the sheet. In order to provide a visual indication of the transmission of current through the CEC a nickel-chromium wire was inserted into both sides of the disk which emitted black body radiation as they transmitted 2.5 A through the CEC.

In order to validate the utility of CECs as electrical vias in extensible soft devices, we characterized the conductivity of CEC disks embedded in elastomeric sheets as a function of extension and compression. For these experiments 4 x 34 x $117 \mathrm{~mm}$ elastomeric sheets were fabricated with a CEC disk at their center with a diameter of $8.8 \pm 0.2 \mathrm{~mm}$ and a height of $7.6 \pm$ $0.3 \mathrm{~mm}$ (based on measurements of the dimensions of $\mathrm{n}=7$ CEC disks) and whose majority fiber axis was perpendicular to the plane of the sheet. For sheet extension experiments, samples were clamped so that the sheets were extended perpendicular to the majority fiber axis of the CEC disk and parallel to the long axis of the sheet with a distance of $70 \mathrm{~mm}$ between the clamps. The samples were found to be highly extensible with an elongation at break of $429 \pm 12 \%$ ( $n=3$ measurements) resulting from delamination between the elastomer sheet and the CEC disk. This resilience to extension exceeds the requirements of most soft devices. Figure S5 shows that the resistance of a CEC disk does not change during an extension of the sample to a $200 \%$ 
elongation ( $\mathrm{n}=7$ measurements of the change in CEC resistance during extension). Only a small change in resistance, $\geq 0.1 \Omega$, was observed when a single sample was cycled 1000 times to an elongation of $100 \%$ (Figure S6). CEC resistance experiments as a function of compression were conducted by placing the sheet samples between conductive plates and compressing the samples parallel to the majority fiber axis of the CEC disk and perpendicular to the long axis of the sheet. Figure $\mathbf{S 7}$ shows that although the resistance amongst the samples varied during initial loading $\left(<13 \mathrm{mN} / \mathrm{mm}^{2}\right)$, this variation reduced at higher loading. A CEC

resistance of $<1.0 \Omega$ was achieved for all samples at a compressive loading of $>13 \mathrm{mN} / \mathrm{mm}^{2}$ ( $\mathrm{n}=$ 7 measurements of the change in CEC resistance during compression).

\subsection{Simple electrical circuits involving non-planar surfaces}

We used the many useful characteristics (e.g., chemistry, flexibility, extensibility, conductivity, etc.) of the composite to achieve function that would be difficult to achieve with other materials (Figure 3) including serpentine wires that are more sophisticated but more difficult to fabricate. The composite can i) be fabricated and subsequently integrated with other elastomeric polymer components; ii) function as an electrical conduit through an insulating elastomeric sheet without significantly perturbing the yield stress of the sheet; iii) be stretched, compressed, bent and folded to make conformal, electrical contact with non-planar surfaces; iv) provide large-area and reconfigurable contact pads; v) combine electrical and mechanical functions. 


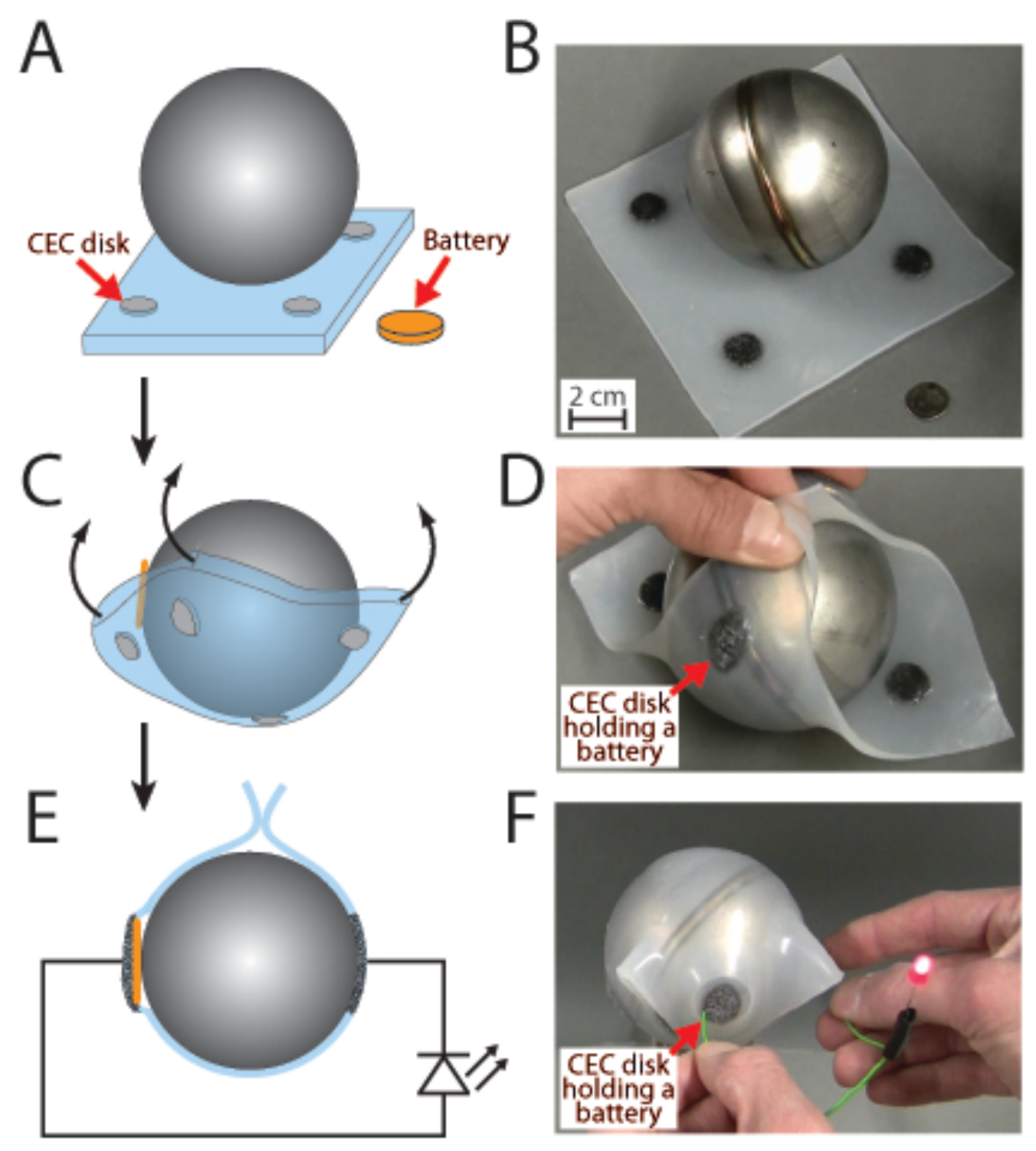

Figure 3. CEC disks embedded in elastomeric sheets can be used to create electrical connections with non-planar surfaces. Here, a CEC containing sheet is simultaneously used as a battery receptacle and as an electrical connection to a metal ball. (A, B) Illustration (left) and photo (right) of a metal sphere, button battery, and an elastomeric sheet with four embedded CEC disks. (C, D) Stretching of the sheet to make conformal contact between the composite and a steel ball. Here the battery is pinned between one of the disks and the ball. (E, F) Circuit diagram and photo of an LED which is illuminated by bridging two of the CEC disks embedded in the sheet. 


\section{WILEY-VCH}

As an example, we made four CEC disks, and embedded them in an elastomeric sheet that we subsequently stretched around a metallic sphere (Figure 3A to 3D). These CEC disks created electrical pathways across the elastomeric sheet that made conductive contact with the sphere at the positions of the disks. We oriented the majority fiber-axis of the disks perpendicular to the surface of the elastomeric sheet in order to minimize electrical resistance across the sheet, and to minimize the stiffness of the CEC in the plane of the sheet. The stress associated with stretching the sheet around the sphere "held" the CEC disks in conformal, electrical contact with the surface of the sphere and could be used to position other objects around the sphere - in this case a button battery (Figure 3C and 3D). Positioning the battery between a conductive CEC disk and the sphere allowed us to illustrate the conductivity of the CEC by completing the circuit with a light-emitting diode (LED, Figure 3E, 3F and Video S4).

\subsection{Connecting hard and soft electrical components}

We can use CECs to create electrical connections to hard electrical components. This capability is useful for connecting conventional hard electrical components (e.g. wires, LEDs, circuits, heating elements, sensors, etc.) to electrical components embedded in soft machines. For example, CEC wires could be fabricated into the body of a soft, pneumatically actuated, legged robot in order to connect the battery, solenoid valves, and pneumatic pump required for operating the robot. We made a prototype demonstrating this capability by fabricating the soft analog of a solderless breadboard (Figure 4). The soft, solderless breadboard was made entirely of a silicone elastomer and steel wool. It consisted of a set of CEC pegs for forming electrical connections to hard electrical components, and CEC fiber bundles connecting selected pegs to create the pattern of connectivity shown in Figure 4A. Electrical connections were 


\section{WILEY-VCH}
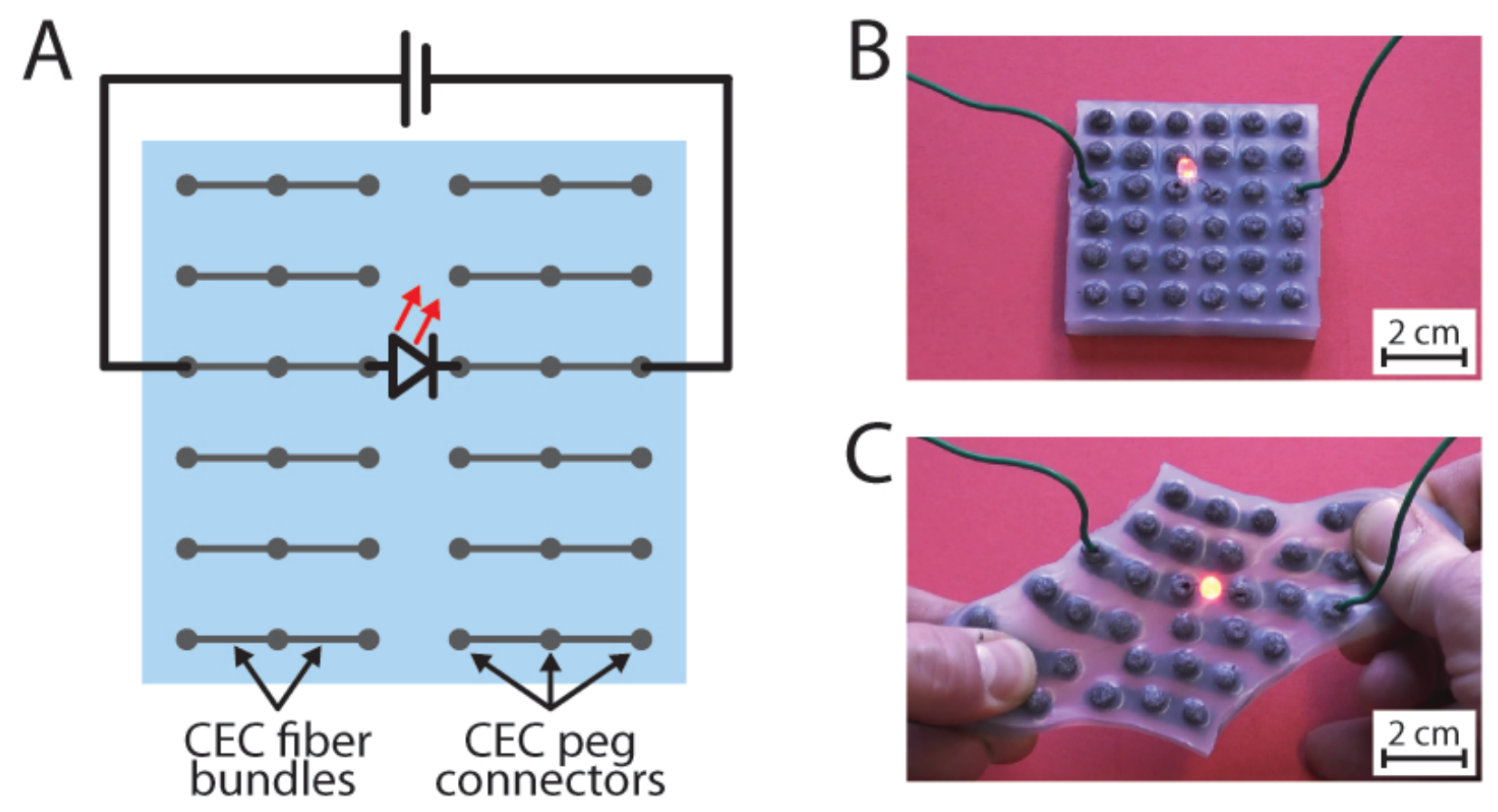

Figure 4. CECs can be used to interface soft and hard electrical components. (A) Circuit diagram of a set of forward biased wires illuminating a LED via electrical connections made entirely by the CEC fiber bundles and receptacles of a soft, solderless breadboard. (B) Photo of a soft, solderless breadboard with a LED and wires inserted into its receptacles to create a closed circuit as evidenced by the illumination of the LED. (C) Photo of the soft, solderless breadboard maintaining its electrical connections during elongation as evidenced by the illumination of the LED. 


\section{WILEY-VCH}

formed by puncturing the pegs of the soft, solderless breadboard with the metal wires of a hard device. The act of puncturing a peg created a permanent, mechanically robust receptacle in the peg for mating hard components. The soft, solderless breadboard had a resistance between adjacent electrically connected pegs of $1.0 \pm 0.4 \Omega(n=24$ measurements of peg to peg resistance) which was sufficiently low to function as wires in a basic circuit. We used this breadboard to connect an LED to a set of electrically biased copper wires via a CEC connection (Figure 4A and 4B). The illumination of this LED established that electrical contact between soft and hard components was maintained when the soft, solderless breadboard was stretched and flexed (Figure 4C and Video S5).

\subsection{Soft touch sensor}

A large portion of the research conducted in the field of soft robotics has focused on pneumatically driven soft actuators, ${ }^{[2,7]}$ creating a need for stretchable electrical connections that can span pressurized interfaces. CECs can be used to create these electrical connections. We demonstrated this capability by making a soft touch sensor (Figure 5A) that closes a circuit when depressed. In this demonstration, the square shape of these CECs was formed by exploiting the attraction of ferromagnetic steel wool to a magnet with a square cross section (Figure S11). The soft touch sensor was fabricated by forming an air-tight seal between two elastomeric sheets with CEC pads at their center. After fabrication, the interior of the switch was pressurized ( $\sim 115 \mathrm{kPa}$ absolute pressure) by a compressed air line seen to the left of each photo and the CECs were punctured with wires to connect the switch in series with the LED circuit. In the absence of an external force (Figure 5B), the pressure kept the CEC pads and 


\section{WILEY-VCH}

A
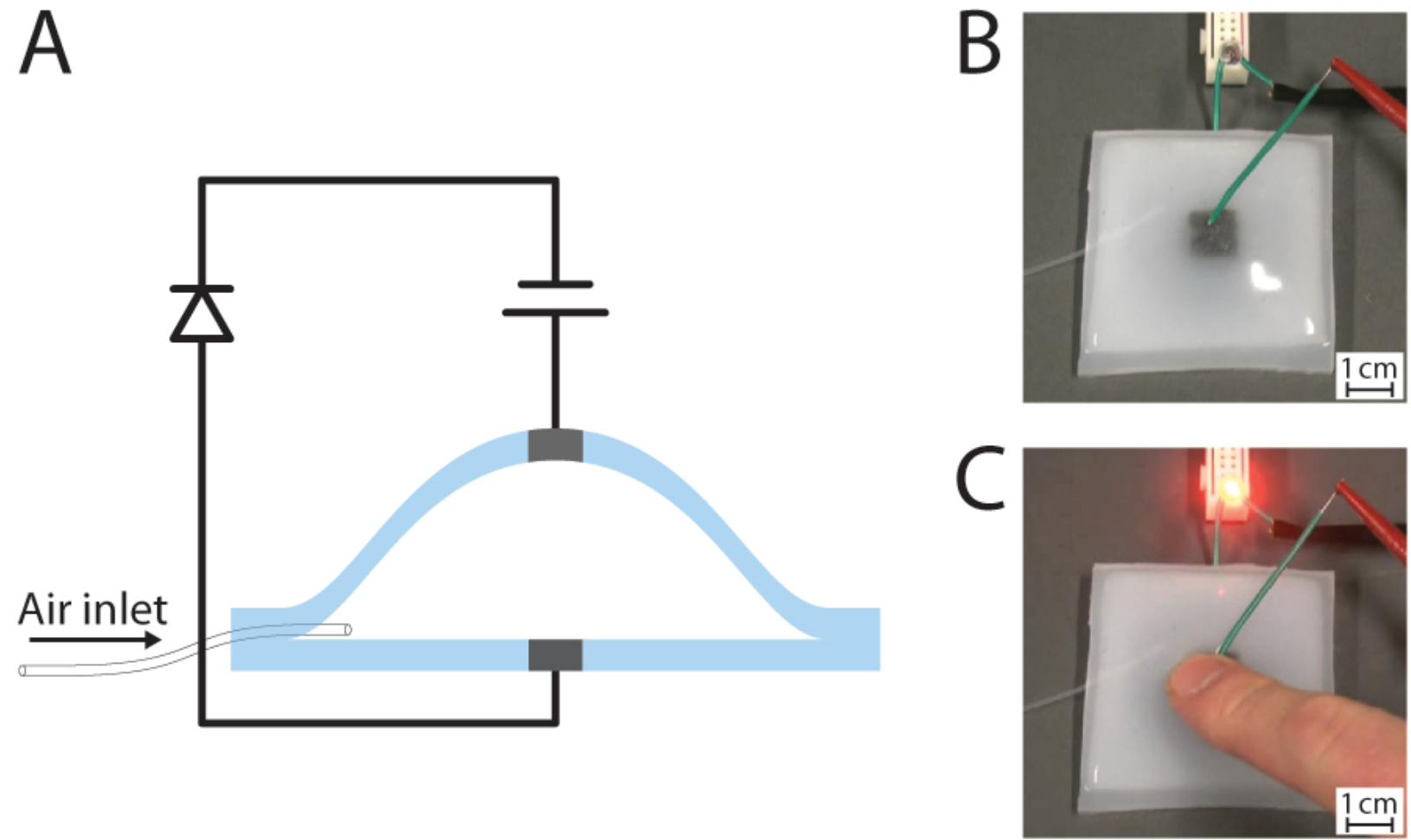

Figure 5. CECs can be used to make a touch sensor. (A) Illustration of a soft touch sensor connected to a simple LED circuit. Here the soft touch sensor consists of a pair of elastomeric sheets, with embedded CEC pads, separated by a volume of pressurized air ( $115 \mathrm{kPa}$ absolute pressure). (B) Photograph of the open circuit with fully separated CEC pads. (C)

Photograph of the system with the top sheet depressed. The circuit is closed, and the LED illuminates. 


\section{WILEY-VCH}

elastomeric sheets separated. When the top sheet was compressed (Figure 5C and Video S6) the pads came into contact, closed the circuit, and caused the LED to illuminate.

\subsection{Soft strain gauge}

The composites make leak-resistant electrical connections to channels containing embedded liquids or greases. Figure 6A shows a soft strain gauge. For this device, we deposited, through extrusion printing, a fine bead of carbon grease onto a thin, elastomeric sheet and brought this bead into contact with bundles of steel wool, the entirety of which was then coated with more elastomer (Figure S12). Using this technique, we were able to create a seamless electrical connection that resisted leaking. This solved a problem often encountered when affixing hard caps to the ends of liquid-filled elastomeric tubes or when inserting conventional wires into liquid filled microfluidic channels. Since this device detects changes in the strain state of the sensor by measuring changes in device resistance, we chose to leave the sections of steel wool that are connected to the ohm meter uncoated by elastomer. Constructing the sensor in this way improves sensor performance by reducing measurement artifacts resulting from changes in the connection between the ohm meter and the CEC's during sensor operation. Video S7 shows the increase in resistance of the device in Figure 6A when strained. This device possessed a sufficient dynamic range for use as a strain sensor in many circuits with a resistance of $10.4 \pm 0.5 \mathrm{k} \Omega$ in its relaxed state and $134.0 \pm 4.9 \mathrm{k} \Omega$ at a strain of $1.2(\mathrm{n}=40$ measurements of the resistance for both the relaxed and extended state). The change in resistance of the carbon grease channel - measured by an ohm meter connected to the CECs at both ends of the device - arises from the thinning and elongation of the bead of grease during strain. Figure $6 \mathrm{~B}$ and $6 \mathrm{C}$ provide an illustration of the relaxed and extended state of 


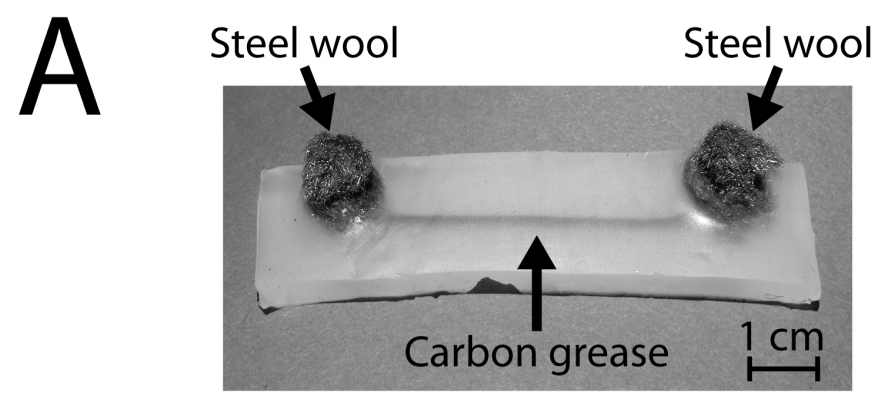

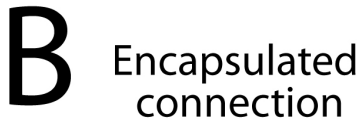
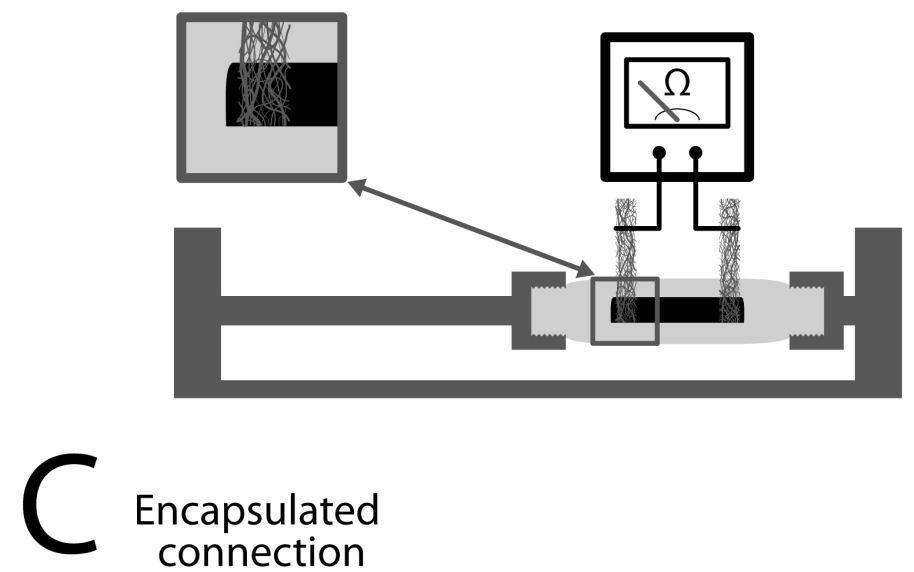

\section{Encapsulated}

connection

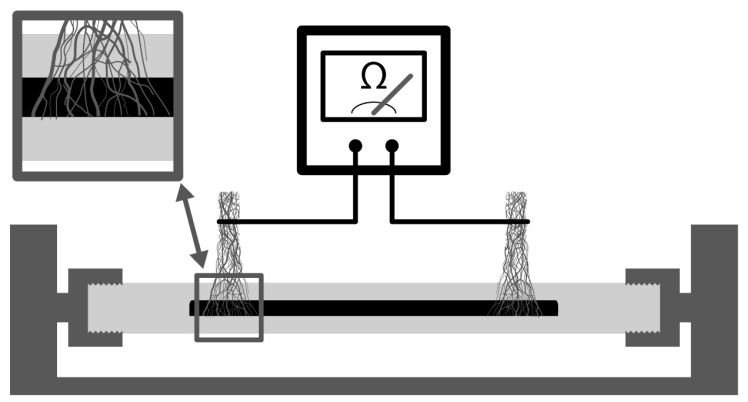

Figure 6. CECs can be used to make leak resistant electrical connections to embedded liquid and grease filled channels. (A) Photograph of a soft strain gauge consisting of an elastomer embedded carbon grease filament contacted at both ends by CEC fiber bundles. (B) Illustration of a soft strain gauge connected to an ohm meter in its low resistance state prior to elongation. (C) Illustration of the soft strain gauge in its high resistance state after thinning and elongation of the carbon grease filament. The enlarged images on the left side of B and C show an elastomer encapsulated CEC electrical connection in its relaxed and elongated states, respectively. 


\section{WILEY-VCH}

the soft strain gauge. The illustration highlights the spreading of the steel fibers of the CEC electrical connection upon strain which is observed in Video S7. This spreading of the steel fibers allows the CECs to maintain electrical contact with the carbon grease channel over repeated cycles of strain without delaminating from the device, as would occur with a monolithic wire. The ability to make leak-resistant electrical connections with CECs will prove useful for creating fluid based sensors for soft robots. For example, by integrating the sensor shown here into a soft actuator, one could obtain real time measurements of the actuators morphology. This data would be invaluable for creating a closed loop control system that orchestrates the motion of a soft robot.

\section{Conclusions}

We have fabricated an elastically-deformable, conductive composite using inexpensive, readily available materials (elastomers and metal wools) and simple procedures. CECs offer low resistance to electrical current and can be easily interfaced/built into structures fabricated from elastomeric polymers, enabling capabilities that are difficult to achieve with conventional electronics. Unlike conventional wires, CECs can be used to distribute electrical current and potential throughout the body of a soft device as the device is compressed and stretched during operation. We have shown that a CEC's combination of conductivity, deformability, and conformability allows for the creation of electrical connections to hard metal components and liquids while they move and change shape. These properties facilitate the interfacing of conventional hard electrical components with soft devices as well as providing a simple means for the construction of completely soft electrical devices. We have focused on CECs made from silicone elastomers and steel wool, but other combinations of elastomers and metal wools 


\section{WILEY-VCH}

are equally compatible with this approach. CECs do not approach the sophistication demonstrated in serpentine interconnects or buckled film based stretchable electronics, where small size is critical to their applications, but CECs are easier to fabricate and solve a common problem in the field of soft devices: viz, how to create an electrical connection through an elastomeric polymer. We believe that CECs will be useful in soft robotics, ${ }^{[2,7]}$ where making stretchable electrical connections through elastomers will facilitate the incorporation of hard electrical and mechanical components (e.g. pneumatic and hydraulic pumps, solenoid valves, nitinol actuators, sensors, etc.) into soft actuators. The fusion of soft actuators with electrical components like CECs, designed for incorporation into soft devices, is an important step on the path to sophisticated soft robots that can sense and respond to their external environment.

\section{Supporting Information}

Supporting Information is available from the Wiley Online Library or from the Whitesides group website (http://gmwgroup.harvard.edu/pubs).

\section{Acknowledgments}

The work presented in this paper was funded by the U.S. Department of Energy, Division of Materials Sciences and Engineering, under award \# ER45852. Partial salary support for CK and SM was provided by the Materials Science and Engineering Center under NSF award \# DMR-0820484. Salary support for AT was provided by the Wyss Institute for Biologically Inspired Engineering at Harvard University. We would like to thank Volume Graphics for their assistance in analyzing the micro X-ray computed tomography data. We would also like to 
thank David Kolesky of the Jennifer Lewis Group, Harvard University, for assistance with the fabrication of the soft strain gauge.

\section{References}

[1] Z. Suo, MRS Bulletin 2012, 37, 218; S. Wagner, S. Bauer, MRS Bulletin 2012, 37, 207; F. Iida, C. Laschi, Procedia Comput. Sci. 2011, 7, 99; M. L. Hammock, A. Chortos, B. C. K. Tee, J. B. H. Tok, Z. Bao, Adv. Mater. 2013, 25, 5997; S. Bauer, S. Bauer-Gogonea, I. Graz, M. Kaltenbrunner, C. Keplinger, R. Schwödiauer, Adv. Mater. 2014, 26, 149.

[2] F. Ilievski, A. D. Mazzeo, R. F. Shepherd, X. Chen, G. M. Whitesides, Angew. Chem. Int. Ed. Engl. 2011, 50, 1890.

[3] C. Keplinger, T. Li, R. Baumgartner, Z. Suo, S. Bauer, Soft Matter 2012, 8, 285.

[4] C. Keplinger, J.-Y. Sun, C. C. Foo, P. Rothemund, G. M. Whitesides, Z. Suo, Science 2013, 341, 984 .

[5] G. G. L. Huai-Ti Lin, Barry Trimmer, Bioinspir. Biomim. 2011, 6; S. Sangok, C. D. Onal, R. Wood, D. Rus, S. Kim, "Peristaltic locomotion with antagonistic actuators in soft robotics", presented at IEEE Int. Conf. Robot. Autom. 2010, 3-7 May 2010, 2010; P. Walters, D. McGoran, NIP \& Digital Fabrication Conference 2011, 2011, 185; J. Zang, S. Ryu, N. Pugno, Q. Wang, Q. Tu, M. J. Buehler, X. Zhao, Nat. Mater. 2013, 12, 321; S. Rosset, H. Shea, Appl. Phys. A 2013, 110, 281.

[6] E. Palleau, D. Morales, M. D. Dickey, O. D. Velev, Nat. Commun. 2013, 4.

[7] R. V. Martinez, J. L. Branch, C. R. Fish, L. Jin, R. F. Shepherd, R. M. D. Nunes, Z. Suo, G. M. Whitesides, Adv. Mater. 2013, 25, 205; R. F. Shepherd, F. Ilievski, W. Choi, S. A. Morin, A. A. Stokes, A. D. Mazzeo, X. Chen, M. Wang, G. M. Whitesides, Proc. Natl. Acad. Sci. USA 2011; R. F. Shepherd, A. A. Stokes, R. M. D. Nunes, G. M. Whitesides, Adv. Mater. 2013, 25, 6709 .

[8] S. Wakimoto, K. Ogura, K. Suzumori, Y. Nishioka, "Miniature soft hand with curling rubber pneumatic actuators", presented at IEEE Int. Conf. Robot. Autom. 2009, 12-17 May 2009, 2009.

[9] S. I. Koichi Suzumori, Hirohisa Tanaka, IEEE ICRA Sacramento, CA 1991, 1, 1622.

[10] T. N. Norihiko Saga, Smart Mater. Struct. 2004, 13, 566.

[11] O. Graudejus, B. Morrison, C. Goletiani, Z. Yu, S. Wagner, Adv. Funct. Mater. 2012, 22, 640 .

[12] I. M. Graz, D. P. J. Cotton, A. Robinson, S. P. Lacour, Appl. Phys. Lett. 2011, 98; S. P. Lacour, C. Tsay, S. Wagner, IEEE Electron Device Lett. 2004, $25,792$.

[13] S. P. Lacour, J. Jones, S. Wagner, T. Li, Z. Suo, P. IEEE 2005, 93, 1459.

[14] H. N. Tsuyoshi Sekitani, Hiroki Maeda, Takanori Fukushima, Takuzo Aida, Kenji Hata, Takao Someya, Nat. Mater. 2009, 8, 494.

[15] T. Sekitani, Y. Noguchi, K. Hata, T. Fukushima, T. Aida, T. Someya, Science 2008, 321, 1468 .

[16] M. Kaltenbrunner, G. Kettlgruber, C. Siket, R. Schwödiauer, S. Bauer, Adv. Mater. 2010, 22, n/a; G. Kettlgruber, M. Kaltenbrunner, C. M. Siket, R. Moser, I. M. Graz, R. Schwodiauer, S. Bauer, J. Mater. Chem. A 2013, 1, 5505. 
[17] S. Xu, Y. Zhang, J. Cho, J. Lee, X. Huang, L. Jia, J. A. Fan, Y. Su, J. Su, H. Zhang, H. Cheng, B. Lu, C. Yu, C. Chuang, T.-i. Kim, T. Song, K. Shigeta, S. Kang, C. Dagdeviren, I. Petrov, P. V. Braun, Y. Huang, U. Paik, J. A. Rogers, Nat. Commun. 2013, 4, 1543.

[18] T. Someya, Y. Kato, T. Sekitani, S. Iba, Y. Noguchi, Y. Murase, H. Kawaguchi, T. Sakurai, Proc. Natl. Acad. Sci. USA 2005, 102, 12321.

[19] D. J. Lipomi, M. Vosgueritchian, B. C. K. Tee, S. L. Hellstrom, J. A. Lee, C. H. Fox, Z. Bao, Nat. Nano. 2011, 6, 788.

[20] C. Yan, J. Wang, X. Wang, W. Kang, M. Cui, C. Y. Foo, P. S. Lee, Adv. Mater. 2014, $26,943$.

[21] B.-R. C. Yong-Lae Park, Robert J.Wood, IEEE Sens. J. 2012, 12, 2711; M. D. Dickey, R. C. Chiechi, R. J. Larsen, E. A. Weiss, D. A. Weitz, G. M. Whitesides, Adv. Funct. Mater. 2008, 18, 1097; S. Zhu, J.-H. So, R. Mays, S. Desai, W. R. Barnes, B. Pourdeyhimi, M. D. Dickey, Adv. Funct. Mater. 2012, n/a; E. Palleau, S. Reece, S. C. Desai, M. E. Smith, M. D. Dickey, Adv. Mater. 2013, n/a.

[22] S. Rosset, M. Niklaus, P. Dubois, H. R. Shea, Adv. Funct. Mater. 2009, 19, 470.

[23] M. G. Urdaneta, R. Delille, E. Smela, Adv. Mater. 2007, 19, 2629.

[24] T. Vervust, F. Bossuyt, F. Axisa, J. Vanfleteren, Mater. Res. Soc. Symp. Proc. 2010, 1271E.

[25] S. Araby, L. Zhang, H.-C. Kuan, J.-B. Dai, P. Majewski, J. Ma, Polymer 2013, 54, 3663; G. Ausanio, A. C. Barone, C. Campana, V. Iannotti, C. Luponio, G. P. Pepe, L. Lanotte, Sensor. Actuat. A-Phys. 2006, 127, 56; K. D. D Bloor, P J Hands, P Laughlin, D Lussey, J. Phys. D: Appl. Phys. 2005, 38, 2851; L. Bokobza, Polymer 2007, 48, 4907; K.-Y. Chun, Y. Oh, J. Rho, J.H. Ahn, Y.-J. Kim, H. R. Choi, S. Baik, Nat. Nano. 2010, 5, 853; L. Flandin, A. Chang, S. Nazarenko, A. Hiltner, E. Baer, J. Appl. Polym. Sci. 2000, 76, 894; W. Hu, R. Wang, Y. Lu, Q. Pei, J. Mater. Chem. C 2014; J.-C. Huang, Adv. Polym. Tech. 2002, 21, 299.

[26] D. S. Gray, J. Tien, C. S. Chen, Adv. Mater. 2004, 16, 393; Y.-Y. Hsu, M. Gonzalez, F. Bossuyt, J. Vanfleteren, I. De Wolf, IEEE T. Electron Dev. 2011, 58, 2680.

[27] S. Béfahy, S. Yunus, T. Pardoen, P. Bertrand, M. Troosters, Appl. Phys. Lett. 2007, 91; P. Gorrn, W. Cao, S. Wagner, Soft Matter 2011, 7, 7177; J. Jones, S. P. Lacour, S. Wagner, Z. Suo, J. Vac. Sci. Technol. A 2004, 22, 1723.

[28] S. P. Kotha, C. Li, S. R. Schmid, J. J. Mason, J. Biomed. Mater. Res. A 2004, 70A, 514; A. E. Naaman, J. Adv. Concr. Technol. 2003, 1, 241; X. Huang, V. Birman, A. Nanni, G. Tunis, Compos. Part B-Eng. 2005, 36, 73.

[29] Y. Lu, C.-F. Tang, M. A. Wright, J. Appl. Polym. Sci. 2002, 84, 2498.

[30] E. R. J. J. Ferdinand P. Beer, John T. DeWolf, David F. Mazurek, Mechanics of materials, McGraw-Hill Higher Education, Boston 2009. 